\title{
Microprocessor Compatible PWM Generator Implement on FPGA
}

\author{
Bapan Singh, Prashant Kumar Dubey, \\ Arijit Roy \\ Students, ECE Dept. \\ Brainware Group of Institutions \\ Kolkata
}

\author{
Debarshi Datta \\ Assistant Professor \\ ECE Dept. \\ Brainware Group of Institutions \\ Kolkata
}

\begin{abstract}
This paper is concerned about microprocessor compatible PWM generator architecture by using Field Programmable Gate Array (FPGA). In PWM the variation of duty cycle change the width of the pulse. The PWM generator (PWMG) is interfaced to the bus of a microprocessor. The microprocessor initiates PWMG to specify duty cycle. The duty cycle remain unchanged until new data available to the PWMG from microprocessor unit. The output signal of PWMG is logic " 1 " and logic " 0 " for a specific time period. The architecture has been designed with VHDL code and verified using Xilinx ISE Design Suite 14.7. The design is successfully implemented on SPRATAN-6 FPGA board. The operating frequency of this proposed architecture is of 292.650MHz.
\end{abstract}

\section{Keywords}

Duty cycle, Microprocessor, PWM,VHDL, FPGA

\section{INTRODUCTION}

A pulse width modulated (PWM) signal is widely used in industry like DC-DC converters, motor speed controlling, solar tracking, power converter and many more. The very low power loss in the switching devices is the main advantage of PWM. In switch off mode no current is flowing and in on state, there is zero voltage drop across the switch. The power loss which is calculated by the multiplication of both voltage and current is zero for these cases. The proper adjustment of the duty cycle, the PWM also uses in digital domain where $\mathrm{ON} / \mathrm{OFF}$ is natural state.

Pulse width modulation is basically variation of pulse with respect to time. The main parameter of PWM is duty cycle which can be varied according the desired value. The duty cycle (D) can be calculated of the following equation

$$
\mathrm{D}=\frac{T_{\text {ON }}}{T}
$$

Where $\mathrm{T}=T_{\text {ON }}+T_{\text {OFF }}$

The output signal can be obtained by following equation

$$
\text { Output }=(\text { duty cycle }) X \text { (input) }
$$

The motor speed can be controlled by variation of duty cycle. For very short period of PWM signal, the motor's speed is directly related to the signal's duty cycle. On the other contrary, for very large time period the motor's speed is no longer controlled by the average value of the PWM signal. The motor rotates with high speed for PWM signal 1 and stop for 0 PWM signal. Figure 1 shows pulse width modulated signal with different duty cycles.

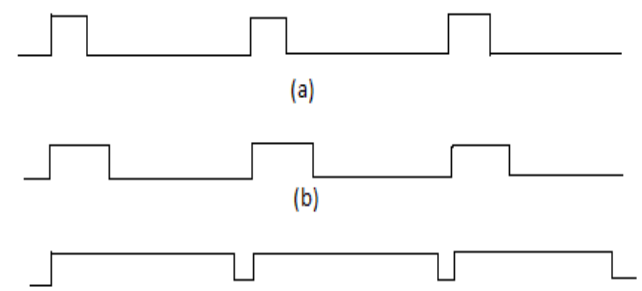

(c)

Figure 1. Pulse width modulated waveforms (a) $20 \%$ duty cycle (b) $40 \%$ duty cycle (c) $90 \%$ duty cycle

FPGAs are one of the suitable platforms for realization PWMG. The inherent parallel data stream, optimization, flexibility and short time to market make it very attractive compare to other processor architectures like DSPs, Microcontrollers. FPGA is the portable platform for several real-world applications.

The implementation of PWM in FPGA is an important research area. The PWM controller was implemented on FPGA with high frequency [1]. In [2] the author proposed a hardware-based PWM signal generation for control in motor. In paper [3], the author described generation of PWM signals with variable duty cycle. The model was codded in VHDL with operating frequency $10 \mathrm{MHz}$ and tested on FPGA. In paper [4], the authors proposed generation of PWM signals using VHDL based on FPGA. The output of PWM signals have a fixed frequency $(11.8 \mathrm{KHz})$ depended on the frequency of sawtooth, and a variable duty cycle that changes from $0 \%$ to $100 \%$.

This paper designed microprocessor compatible PWM signal generation in VHDL and successfully tested on FPGA board.

\section{PROPOSED ARCHITECTURE}

This section is been designed PWM signal generator (PWMSG) to be interfaced to the bus of a microprocessor. The proposed design is consisted of register and counter with proper data input. The microprocessor writes a data byte to the PWMSG for generating specific duty cycle. The output of

PWMSG duty cycle is unchanged till the microprocessor writes a new data byte to the PWMSG. The block diagram of the proposed system is shown in Figure 2. The microprocessor clock (clk) is used to operate the clock input of the PWMSG. The microprocessor clock frequency is reduced by prescalar 
subsystem to generate low frequency signal, ps. This ps signal is applied to the period counter to count the triggering edge of the microprocessor clock. The period counter output (period_cnt) is used for period of PWM signal which is 256 times period of the prescalar output (ps). The period of the PWM output signal, pwm_out, is defined as the time require period_cnt to cycle from 0 through 255 and then roll over to 0 . The subsystem duty cycle provides 8-bit duty_cycle. The microprocessor is written on duty cycle subsystem to control the duty_cycle. The microprocessor provides data to duty cycle subsystem and controls the write operation using the cs_bar and we_bar inputs. The value of pwm_out duty cycle is depend upon data input the duty cycle subsystem divided by 256.

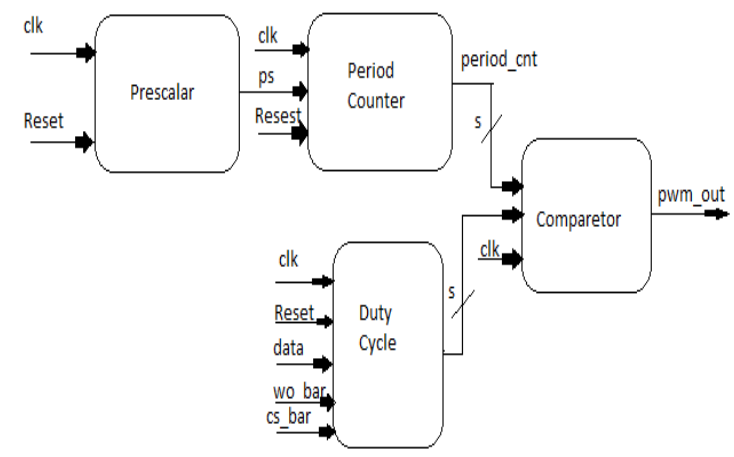

Figure 2. Microprocessor compatible Pulse Width Modulated Signal Generator

For example, to achieve $25 \%$ duty cycle the data input from microprocessor is of 64 to duty cycle subsystem. Then comparator compares the output of the period counter period_cnt with the duty_cycle from the duty cycle subsystem. If duty_cycle value is more than period_cnt, then output of the comparator pwm_out ' 1 '; otherwise ' 0 '.

\section{FPGA IMPLEMENTATION}

This proposed hardware modules has been simulated along with its RTL schematics by Xilinx ISE design suite 14.7 and synthesized bits stream are downloaded on Spartan 6 to target device XC6SLX45. VHDL code for proposed design is given below..

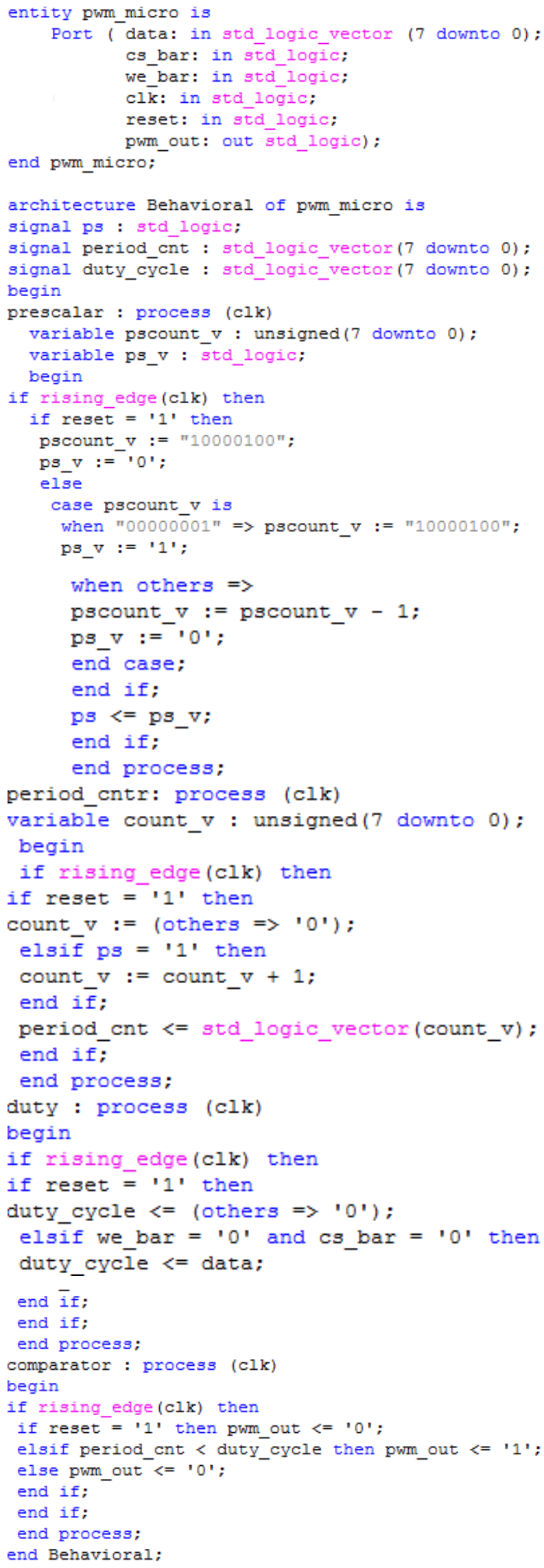


The simulated waveform is shown in Figure 3.

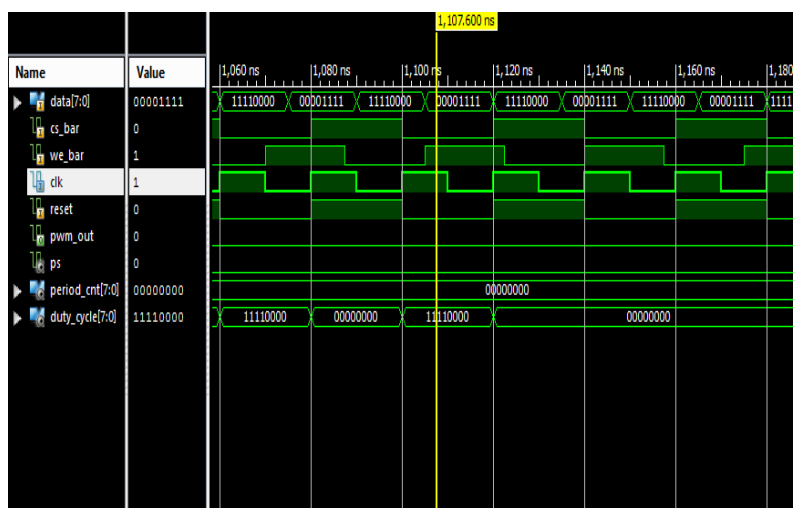

Fig 3: PWMG Timing waveform

The RTL schematic of the PWMSG is shown in Figure 4.

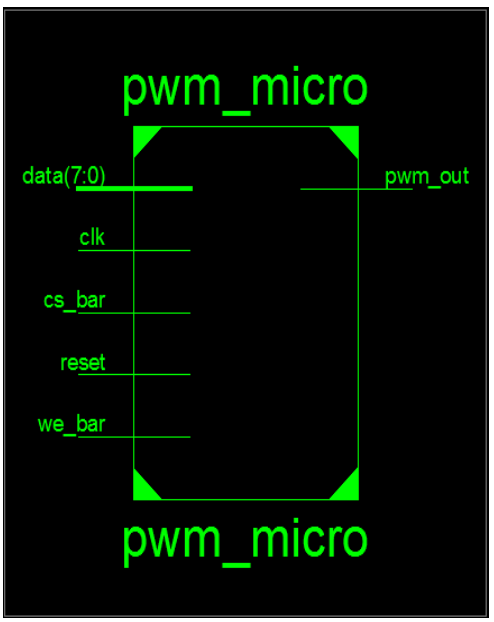

Fig 4: RTL Schematic of PWMG

The hardware resources like slice registers, LUTs, flip-flops are described in table 1. The operating speed of this PWMSG is of $292.650 \mathrm{MHz}$.

Table Error! No sequence specified.. Device utilization summary of PWMG

\begin{tabular}{|c|c|}
\hline Synthesis Parameter & PWM Generator \\
\hline Number Slice Registers & 26 \\
\hline Number of Slice LUTs & 24 \\
\hline Number used as Flip-Flops & 26 \\
\hline Number used as logic & 23 \\
\hline Number of IOBs & 13 \\
\hline
\end{tabular}

\section{CONCLUSION}

In this paper, FPGA based microprocessor compatible PWM signal generation is discussed. Microprocessor writes data into PWMG to produce particular duty cycle. Microprocessor controls the PWMG for providing variation pulse width. The system has been designed in VHDL and functional verification is obtained from Xilinx ISE 14.7 simulator. The synthesized bit stream is downloaded on Spartan-6 FPGA board to yield hardware resources and computation time. The experimental result shows that proposed designed occupy less number of slices which represent the area of PWMG. The operating frequency of the PWMG is of $292.650 \mathrm{MHz}$ which leads to fastest operation. The output of the PWM signal is applicable to DC-DC converter, motor, robot and many other applications.

\section{ACKNOWLEDGMENTS}

Thanks to Electronics and Communications engineering department of Brainware Group of Institutions for providing SPARTAN -6 FPGA board.

\section{REFERENCES}

[1] S. Kirnapure, V. R. Wadhankar, "Review on Design of PWM Controller Using FPGA," International Journal of Science and Research (IJSR), Volume 4 Issue 4, April, pp. 1489-1492, 2015.

[2] R. Baby, "FPGA Based Digital Pulse Width Modulation Using Xilinx DCM for Motor Controlling”, International Journal of Engineering Research \& Technology (IJERT), ISSN: 2278-0181, Vol. 3 Issue 3, March - 2014.

[3] Sunita,R Srinivasan,Ramsagar, "Generation of Variable Duty cycle PWM using FPGA”, IOSR Journal of VLSI and Signal processing (IOSR,JVSP)Vol-4,Issue 6,VER.II,PP 01-03 e-ISSN:2319-42100,p-ISSN No.:2319-4197, Nov-Dec.2014.

[4] Z. Nouman, B. Klima, J. Knobloch, "Generating PWM Signals With Variable Duty From 0\% to 100\% Based FPGA SPARTAN3ANA", Electrorevue ISSN 12131539 Vol..4, pp 75-80, Dec. 2013.

[5] Kurt wick, "Pulse width Modulation \& FPGA", University of Minnesota, AAPT Philadelphia 2012.

[6] Y. F. Liu, P. C. Sen; "Digital control of switching power converters", in Proc. IEEE Control Application Conf. (CCA”05), pp.635-640, 2005.

[7] Ericsson, "Power Supply goes Digital", white paper,Oct2006.

[8] Application Report, "Designing a TMS320F280x Based Digitally Controlled Dc-Dc Switching Power Supply", Texas Instrument, July 2005.

[9] W. Wayne, "FPGA -Based System Design", Pearson Education, Inc, U.S.A, 2004.

[10] Gwaltney, "FPGA Implementation of controls", 2003. 